EPJ Web of Conferences 38, 01001 (2012)

DOI: $10.1051 /$ epjconf/20123801001

(c) Owned by the authors, published by EDP Sciences, 2012

\title{
Processes in massive nuclei reactions and the way to complete fusion of reactants. What perspectives for the synthesis of heavier superheavy elements?
}

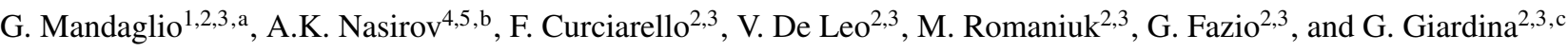 \\ 1 Centro Siciliano di Fisica Nucleare e Struttura della Materia, 95125 Catania, Italy \\ 2 Dipartimento di Fisica e di Scienze della Terra dell'Universitá di Messina, 98166 Messina, Italy \\ 3 Istituto Nazionale di Fisica Nucleare, Sezione di Catania, 95123 Catania, Italy \\ 4 Joint Institute for Nuclear Research, 141980 Dubna, Russia \\ 5 Institute for Nuclear Physics, 100214 Tashkent, Uzbekistan
}

\begin{abstract}
By using the dinuclear system (DNS) model we determine the capture of reactants at the first stage of reaction, the competition between the DNS decay by the quasifission $(\mathrm{QF})$ and the complete fusion $(\mathrm{CF})$ process up to formation of the compound nucleus $(\mathrm{CN})$ having compact shape. Further evolution of the $\mathrm{CN}$ is considered as its fission into two fragments or formation of evaporation residues (ER) by its cooling after emission of neutrons or/and charged light particles. Disappearance of the $\mathrm{CN}$ fission barrier due to its fast rotation leads to the fast fission (FF) by formation of fissionlike fragments. The results of calculations for the mass symmetric ${ }^{136} \mathrm{Xe}+{ }^{136} \mathrm{Xe}$ reaction, almost mass symmetric ${ }^{108} \mathrm{Mo}+{ }^{144} \mathrm{Ba}$ reaction, and mass asymmetric like ${ }^{24} \mathrm{Mg}+{ }^{238} \mathrm{U}$ and ${ }^{34} \mathrm{~S}+{ }^{248} \mathrm{Cm}$ reactions are discussed. The fusion probability $P_{\mathrm{CN}}$ calculated for many massive nuclei reactions leading to formation of superheavy nuclei have been analyzed. The reactions which can lead in perspective to the synthesis of superheavy elements in the $Z=120-126$ range and, eventually, also to heaviest nuclei, are discussed.
\end{abstract}

\section{Introduction}

The analysis of reaction dynamics and competition between the processes which take place at various steps of reaction with massive nuclei can explain why it is practically improbable to reach the stage of $\mathrm{CN}$ in many reactions: we will discuss as an example the case of ${ }^{136} \mathrm{Xe}+{ }^{136} \mathrm{Xe}$ reaction. The study of the hypothetical collision between the ${ }^{108} \mathrm{Mo}$ and ${ }^{144} \mathrm{Ba}$ nuclei allows us to demonstrate why the fusion of massive reactants is not the inverse process of fission with almost symmetric fragments which are formed in the case of the ${ }^{252} \mathrm{Cf}$ spontaneous fission. The failure of many experiments is connected not only with the difficulties in the measurement of the evaporation residue cross sections which are lower than $0.5 \mathrm{pb}$ but also in the inadequacy of estimation of the complete fusion probability [1-3]. The reported difficulties are related not only with the theoretical estimation of the complete fusion and evaporation residue cross section but also in the not univocal experimental identification of fusion-fission fragments among the quasifission and fast fission fragments. We will discuss the limits of reaching $\mathrm{CN}$ with charge heavier than $Z=120$ due to the dominant repulsive Coulomb effects and strong centrifugal forces in very massive nuclei reactions. The partial fusion cross sections present the effect of the angular momentum on the intrinsic fusion barrier and quasifission barrier, and the effect of the angles of the symmetry axes of the two deformed projectile and target

\footnotetext{
a e-mail: gmandaglio@unime.it

b e-mail: nasirov@jinr.ru

c e-mail: ggiardina@unime.it
}

nuclei relative to the beam direction on the Coulomb barrier values and depth of potential well in nucleus-nucleus interaction. It will be shown that the evaporation residue cross sections decrease by increasing the angular momentum due to its influence on the fission barrier of the rotating CN.

In order to give realistic estimations of cross sections of the reaction products by mass symmetric or almost symmetric entrance channel an adequate model allowing us to describe by a likelihood way the complex dynamics of the mechanisms during all stages of reaction has to be developed. In fact, in the last stage of nuclear reaction, the formed $\mathrm{CN}$ may de-excite by fission (producing fusionfission fragments) or by emission of light particles. The reaction products that survive fission are ER. The registration of ER is clear evidence of the $\mathrm{CN}$ formation, but in case of reactions with massive nuclei, generally, the knowledge about ER's only it is not enough to determine the complete fusion cross section and to understand the dynamics of the de-excitation cascade of $\mathrm{CN}$ if the true fission fragments are not included into consideration. On the other hand, the correct identification of an evaporation residue nucleus by the observation of its $\alpha$-decay chain does not assure if the target material contains other isotopes of the nucleus under consideration. In fact, for example, in the case of the ${ }^{48} \mathrm{Ca}+{ }^{249} \mathrm{Cf}$ reaction, the identification of the ${ }^{294} \mathrm{Hs}$ nucleus as the evaporation residue of the ${ }^{297} \mathrm{Hs} \mathrm{CN}$ after the emission of 3 neutrons (see the experiment reported in Ref. [4]) cannot assure that the collected events corresponding to the ${ }^{294} \mathrm{Hs}$ nucleus are obtained only due to the mentioned reaction leading to the formation of ${ }^{297} \mathrm{Hs} \mathrm{CN}$ because in the interaction of projectile ${ }^{48} \mathrm{Ca}$ with ${ }^{250} \mathrm{Cf}$ nucleus which is 
inevitably present in the target due to the finite resolution of the mass separation. Therefore, it is also necessary to consider the contribution of this reaction to the ER formation. In the last case the ${ }^{48} \mathrm{Ca}+{ }^{250} \mathrm{Cf}$ reaction (leading to the ${ }^{298} \mathrm{Hs} \mathrm{CN}$ ) the same ${ }^{294} \mathrm{Hs}$ evaporation residue nucleus is formed after emission of 4 neutrons from $\mathrm{CN}$. This effect depends on the $E_{\mathrm{CN}}^{*}$ excitation energy of $\mathrm{CN}$ which is determined by the collision energy $E_{\mathrm{c} . \mathrm{m}}^{*}$. In addition, the use of some assumptions about the reaction mechanisms leading to the formation of the observed fissionlike fragments, does not allow for sure correct determination of the fusion-fission contribution in the case of overlapping of the mass fragment distributions of different processes (quasifission, fast fission and fusion-fission). The exigence and importance to have a multiparameter and sensitive model is strongly connected with the requirement to reach reliable results and with the possibility to give reliable estimations of perspectives for the synthesis of superheavy elements. If the estimations reported in figures 3 (a) and (b) of Ref. [5] about evaporation residue cross section after $2 n, 3 n$, and $4 n$ emission which are peaked at about the same $E^{*}=40 \mathrm{MeV}$ of the ${ }^{298} 116$ excitation energy are reliable results, then immediately arises the question: what process and barriers can describe with appreciable probabilities the emission of 2 and 3 neutrons that take away about 43 and $48 \mathrm{MeV}$ (or also more) of excitation energy from the ${ }^{298} 116$ and ${ }^{299} 118$ $\mathrm{CN}$, respectively? These results mean that each neutron in the evaporation of $2 n$ is emitted with a kinetic energy of about 15 times the nuclear temperature of the ${ }^{298} 116 \mathrm{CN}$, and 10 times the nuclear temperature of ${ }^{299} 118 \mathrm{CN}$ in the $3 \mathrm{n}$ evaporation process. That is a fully improbable result.

\section{Model and formalism}

By using the DNS model [6], the first stage of reaction is the capture formation of a DNS after full momentum transfer of the relative motion of colliding nuclei into a rotating and excited nuclear system. In the deep inelastic collisions DNS is formed but the full momentum transfer does not occur. Therefore, the deep inelastic collisions are not capture reactions.

The partial capture cross section at a given energy $E_{\mathrm{c} . \mathrm{m}}$. and orbital angular momentum $\ell$ is determined by formula

$$
\sigma_{\text {cap }}^{\ell}\left(E_{\text {c.m. }}\right)=\pi \boldsymbol{A}^{2} \mathcal{P}_{\text {cap }}^{\ell}\left(E_{\text {c.m. }}\right)
$$

where $\mathcal{P}_{\text {cap }}^{\ell}\left(E_{\text {c.m. }}\right)$ is the capture probability meaning the path of collision is trapped into the well of the nucleusnucleus potential after dissipation of the part of the initial relative kinetic energy and orbital angular momentum [7, 8]. The capture probability $\mathcal{P}_{\text {cap }}^{\ell}$ is equal to 1 or 0 for a given $E_{\text {c.m. }}$ energy and orbital angular momentum $\ell$. Our calculations showed that, depending on the center-of-mass system energy $E_{\text {c.m. }}$, there can be "window" in the orbital angular momentum for capture with respect to the following conditions $[7,8]$ :

$\mathcal{P}_{\text {cap }}^{\ell}\left(E_{\text {c.m. }}\right)= \begin{cases}1, & \text { if } \ell_{\min } \leq \ell \leq \ell_{\mathrm{d}} \text { and } E_{\text {c.m. }}>V_{\text {Coul }} \\ 0, & \text { if } \ell<\ell_{\text {min }} \text { or } \ell>\ell_{\mathrm{d}} \text { and } E_{\text {c.m. }}>V_{\text {Coul }} \\ 0, & \text { for all } \ell \text { if } E_{\text {c.m. }} \leq V_{\text {Coul }} .\end{cases}$

The boundary values $\ell_{\min }$ and $\ell_{d}$ of the partial waves leading to capture depend on the dynamics of collision and they are determined by solving the equations of motion for the relative distance $R$ and orbital angular momentum $\ell$ [911]. At lower energies, $\ell_{\min }$ decreases to zero and we do not observe the $\ell$ "window": $0 \leq \ell \leq \ell_{d}$. The range of the $\ell$ "window" is defined by the size of the potential well of the nucleus-nucleus potential $V\left(R, Z_{1}, Z_{2}\right)$ and the values of the radial $\gamma_{R}$ and tangential $\gamma_{t}$ friction coefficients, as well as by the moment of inertia for the relative motion $[8,10]$.

The quasifission process competes with formation of complete fusion. This process occurs when DNS prefers to break down into two fragments instead of transforming into the fully equilibrated $\mathrm{CN}$.

The fusion excitation function is determined by product of the partial capture cross section $\sigma_{c a p}^{\ell}$ and the fusion probability $P_{C N}$ of DNS, at various $E_{\text {c.m. }}$. values

$$
\begin{array}{r}
\sigma_{\text {fus }}\left(E_{\text {c.m. }} ; \beta_{P}, \alpha_{T}\right)=\sum_{\ell=0}^{\ell_{f}}(2 \ell+1) \times \\
\times \sigma_{\text {cap }}\left(E_{\text {c.m. }}, \ell ; \beta_{P}, \alpha_{T}\right) P_{\mathrm{CN}}\left(E_{\text {c.m. }}, \ell ; \beta_{P}, \alpha_{T}\right) .
\end{array}
$$

Obviously, the quasifission cross section is defined by

$$
\begin{array}{r}
\sigma_{\mathrm{qfis}}\left(E_{\mathrm{c} . \mathrm{m} .} ; \beta_{P}, \alpha_{T}\right)=\sum_{\ell=0}^{\ell_{d}}(2 \ell+1) \times \\
\times \sigma_{\text {cap }}\left(E_{\mathrm{c} . \mathrm{m} .}, \ell ; \beta_{P}, \alpha_{T}\right)\left(1-P_{\mathrm{CN}}\left(E_{\mathrm{c} . \mathrm{m} .}, \ell ; \beta_{P}, \alpha_{T}\right)\right) .
\end{array}
$$

For more specific details and descriptions of the model see Refs.[7, 8, 12-14].

Here, in order to show the sensitivity of our model, in figure 1 , we present the calculated $P_{\mathrm{CN}}$ fusion probability as a function of the orbital angular momentum $\ell(\hbar)$, at excitation energies $E_{\mathrm{CN}}^{*}=49 \mathrm{MeV}$ (dashed line) and $63 \mathrm{MeV}$ (full line) of the ${ }^{202} \mathrm{~Pb} \mathrm{CN}$ which is formed in the ${ }^{48} \mathrm{Ca}+{ }^{154} \mathrm{Sm}$ reaction. The figure shows how much the $P_{\mathrm{CN}}$

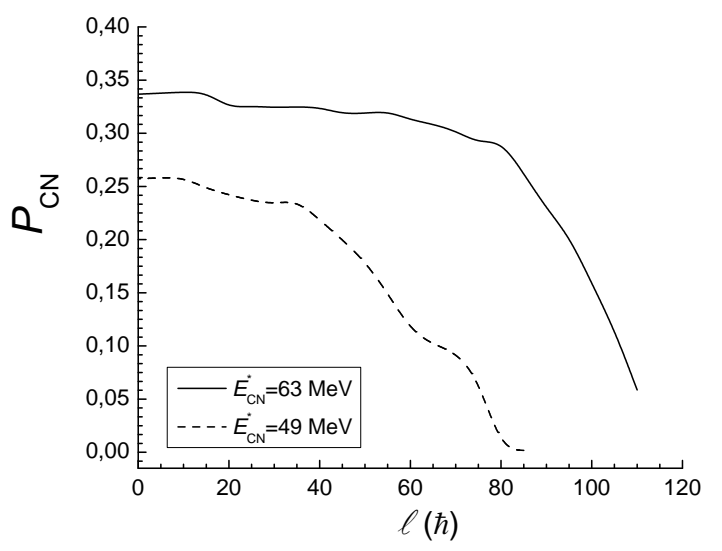

Fig. 1. The $P_{\mathrm{CN}}$ fusion probability calculation versus the orbital angular momentum $\ell(\hbar)$ for the ${ }^{48} \mathrm{Ca}+{ }^{154} \mathrm{Sm}$ reaction, at two different $E_{\mathrm{CN}}^{*}$ values of the ${ }^{202} \mathrm{~Pb} \mathrm{CN}$.

fusion probability changes with the $\ell(\hbar)$ value at a fixed $E_{\mathrm{CN}}^{*}$ excitation energy of $\mathrm{CN}$, and how much does the $P_{\mathrm{CN}}$ trend change at two different $E_{\mathrm{CN}}^{*}$ values. Therefore, the methods that do not take into account in calculation the dependence of the $P_{\mathrm{CN}}$ fusion probability on the collision energy $E_{\text {c.m. }}$, angular momentum $\ell(\hbar)$, and on the orientation angles of the axial symmetry axes of deformed reacting nuclei cannot reach reliable values of the fusion cross section. 
The fast fission cross section is calculated by summing the contributions of the partial waves corresponding to the range $\ell_{f} \leq \ell \leq \ell_{d}$ leading to the formation of a mononucleus where the fission barrier $B_{\mathrm{f}}$ is zero in such range of $\ell$, and the system promptly decays into two fragments

$$
\begin{array}{r}
\sigma_{\text {fastfis }}\left(E_{\text {c.m. }} ; \beta_{P}, \alpha_{T}\right)=\sum_{\ell_{f}}^{\ell_{d}}(2 \ell+1) \times \\
\times \sigma_{\text {cap }}\left(E_{\text {c.m. }}, \ell ; \beta_{P}, \alpha_{T}\right) P_{\mathrm{CN}}\left(E_{\text {c.m. }}, \ell ; \beta_{P}, \alpha_{T}\right) .
\end{array}
$$

The capture cross section in the framework of the DNS model is equal to the sum of the quasifission, fusion, and fast fission cross sections

$$
\begin{array}{r}
\sigma_{\text {cap }}^{\ell}\left(E_{\text {c.m. }} ; \beta_{P}, \alpha_{T}\right)=\sigma_{\text {qfiss }}^{\ell}\left(E_{\text {c.m. }} ; \beta_{P}, \alpha_{T}\right) \\
+\sigma_{\text {fus }}^{\ell}\left(E_{\text {c.m. }} ; \beta_{P}, \alpha_{T}\right)+\sigma_{\text {fastfis }}^{\ell}\left(E_{\text {c.m. }} ; \beta_{P}, \alpha_{T}\right) .
\end{array}
$$

It is clear that the fusion cross section includes the cross sections of evaporation residues and fusion-fission products. The ER cross section is calculated by the advanced statistical code[15-17] that takes into account the damping of the shell correction in the fission barrier as a function of nuclear temperature and orbital angular momentum in determination of the survival probability $W_{\operatorname{sur}(x-1)}\left(E_{x}^{*}, \ell\right)$

$$
\sigma_{\mathrm{ER}(\mathrm{x})}\left(E_{x}^{*}\right)=\sum_{\ell=0}^{\ell_{d}}(2 \ell+1) \sigma_{(x-1)}^{\ell}\left(E_{x}^{*}\right) W_{\mathrm{sur}(x-1)}\left(E_{x}^{*}, \ell\right) .
$$

We able to calculate mass- and angle distribution of quasifission and fusion-fission fragments, anisotropy of the fission fragment angular distribution and the dependence of cross sections, Coulomb barrier, intrinsic fusion barrier and quasifission barrier as a function of the orientation angle of the symmetry axes of colliding nuclei (see Refs. $[14,18]$ ). In figure 2 , for example, we present the mass distribution of quasifission fragments (see figure 2 for the ${ }^{48} \mathrm{Ca}+{ }^{154} \mathrm{Sm}$ reaction). In many cases, in dependence on the entrance channel peculiarities, the mass distributions of fusion-fission, quasifission and the fast fission fragments can overlap $[14,19]$, as a result the real difficulties arise in the analysis of experimental data in order to identify the true yields of fragments according to corresponding processes in heavy ion collisions. Figure 2 shows that at lower $E_{\text {c.m. }}$ energy the mass distribution of quasifission products populate the asymmetric mass region at any lifetime value of DNS (see figure $2(\mathrm{a})$ ), while at higher $E_{\text {c.m. }}$ energy it is also populated the symmetric mass region for longer DNS lifetimes (see figure 2 (b)).

The lifetime of an excited DNS for a given reaction depends on the initial collision energy $E_{\text {c.m. }}$ and angular momentum distribution values [19]. The nuclei constituting the DNS change their mass and charge by nucleon exchange, as well as their shapes during evolution of DNS. The evolution of DNS depends on its excitation energy, orientation angles of the axial symmetry axes and shell structures of reacting nuclei. Therefore, the DNS during its evolution can evolve to complete fusion (fusion process) or can decay into two fragments (quasifission process). The competition between these two processes is related to the values of intrinsic fusion barrier $B_{\text {fus }}^{*}$ and quasifission barrier $B_{\mathrm{qf}}[20-22]$ depending on the peculiarities of reacting nuclei, beam energy and angular momentum distribution.
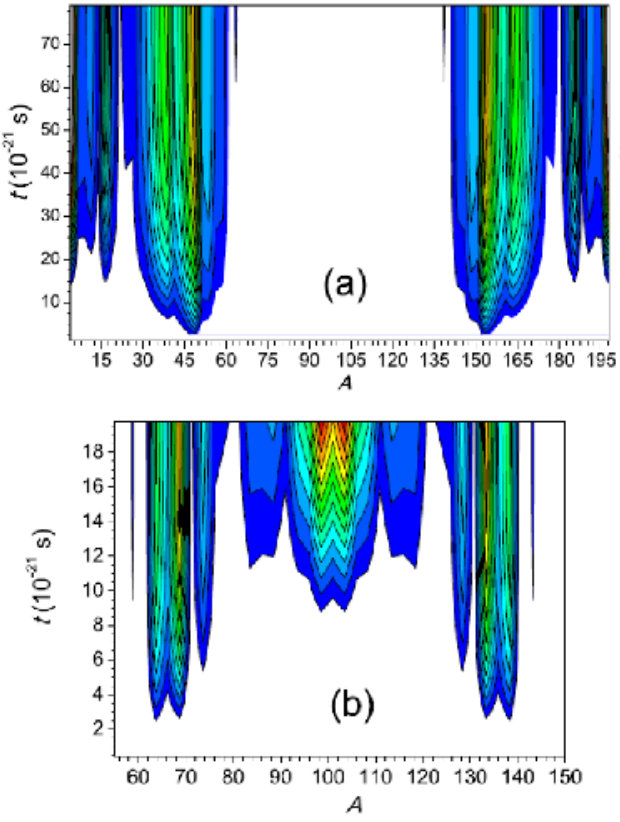

Fig. 2. (a) The mass distribution of the quasifission products yield in the ${ }^{48} \mathrm{Ca}+{ }^{154} \mathrm{Sm}$ reaction at $E_{\text {c.m. }}=140 \mathrm{MeV}$ as a function of the lifetime of DNS formed at capture stage. (b) The mass distribution of the quasifission product yields in the ${ }^{48} \mathrm{Ca}+{ }^{154} \mathrm{Sm}$ reaction at $E_{\mathrm{c} . \mathrm{m} .}=160 \mathrm{MeV}$ as a function of the lifetime of DNS.

\section{Study of the ${ }^{136} \mathrm{Xe}+{ }^{136} \mathrm{Xe}$ and ${ }^{108} \mathrm{Mo}+{ }^{144} \mathrm{Ba}$ reactions}

In order to check, can always any projectile and target combination lead to the complete fusion of reactants (having enough beam energy to overcome the Coulomb barrier) and to synthesize the wanted superheavy element (SHE), as an example, we consider the mass symmetric ${ }^{136} \mathrm{Xe}+$ ${ }^{136} \mathrm{Xe}$ reaction leading to the ${ }^{272} \mathrm{Hs} \mathrm{CN}$. By using the procedure presented in previous Sec. 2, for this reaction we obtain the results which are shown in figure 3. In figure 3, (a) the capture, quasifission, fusion and fast fission cross sections versus $E_{\text {c.m. }}$ energy are reported, while figure 3 (b) shows the fusion probability $P_{\mathrm{CN}}$ in the same explored $E_{\mathrm{c} . \mathrm{m} .}$ energy range. As on can see the capture cross section for the ${ }^{136} \mathrm{Xe}+{ }^{136} \mathrm{Xe}$ reaction is about $10 \mathrm{mb}$ in the explored energy range while the fusion cross section leading to the ${ }^{272} \mathrm{Hs} \mathrm{CN}$ ranges between 0.1 and $10 \mathrm{pb}$ (with a fusion probability of about $10^{-14}-10^{-11}$ ) in the same $E_{\mathrm{c} . \mathrm{m}}$. interval. From our investigations we can conclude that the evaporation residue cross section is much lower than $10^{-10}$ $\mathrm{pb}$. Such a value practically means that no synthesis events of reacting nuclei occur ${ }^{136} \mathrm{Xe}+{ }^{136} \mathrm{Xe}$.

For a comparison with the results of the last reaction, in figure 4 (a), we present the results obtained in this work for the mass asymmetric ${ }^{24} \mathrm{Mg}+{ }^{248} \mathrm{Cm}$ reaction leading to the same ${ }^{272} \mathrm{Hs} \mathrm{CN}$ where at lower-medium $E_{\text {c.m. }}$. energy range the fusion process is dominated in the reaction dynamics, while at high energy the quasifission process prevails. At energies $E_{\text {c.m. }} \approx 135 \mathrm{MeV}$ the fusion-fission cross section is about $150 \mathrm{mb}$ and mass distribution of the symmetric fission fragments are peaked around the mass of the ${ }^{136} \mathrm{Xe}$ nucleus, while the ER cross section is about some pb (see figure 4(b)). From the comparison of the results presented in figures 3 and 4, we can conclude that the fusion- 

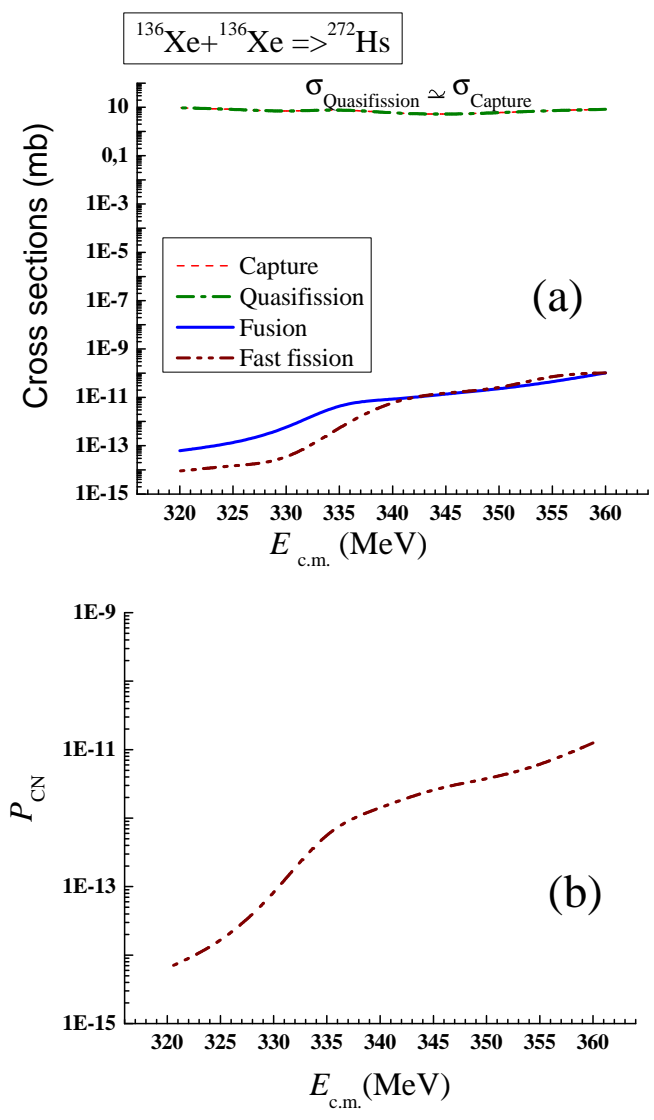

Fig. 3. The capture, quasifission, fusion and fast fission cross sections (panel (a)), and the $P_{\mathrm{CN}}$ fusion probability (panel (b)), versus the $E_{\mathrm{c} . \mathrm{m} .}$ energy of the ${ }^{136} \mathrm{Xe}+{ }^{136} \mathrm{Xe}$ reaction.

fission cross section in the ${ }^{24} \mathrm{Mg}+{ }^{248} \mathrm{Cm}$ reaction at $E_{\mathrm{CN}}^{*}$ of about $55 \mathrm{MeV}$ is about $150 \mathrm{mb}$, while the results for the above-mentioned fusion-fission cross section with the mass symmetric distribution in the ${ }^{136} \mathrm{Xe}+{ }^{136} \mathrm{Xe}$ reaction is about some $\mathrm{pb}$ (because the reaction dynamics is completely dominated by the quasifission process) that is about $10^{-11}$ times lower than the ${ }^{136} \mathrm{Xe}$ fusion-fission fragment yield obtained by the ${ }^{24} \mathrm{Mg}+{ }^{248} \mathrm{Cm}$ reaction. Such a result means that, at the present experimental conditions, it is impossible to register 1 event of synthesis by the ${ }^{136} \mathrm{Xe}+{ }^{136} \mathrm{Xe}$ reaction during 10 billion of years of beam dose. Experimentalists and laboratories are friendly invited to disavow such an estimation.

An analogous consideration attains for the study of the ${ }^{108} \mathrm{Mo}+{ }^{144} \mathrm{Ba}$ reaction in order to obtain the ${ }^{252} \mathrm{Cf} \mathrm{CN}$ by fusion of reactants and to compare such a process - believed as the possible inverse process of the fission - with the ${ }^{252} \mathrm{Cf}$ spontaneous fission leading to the asymmetric mass fragment distribution peaked at ${ }^{108} \mathrm{Mo}$ (for the light fragment) and ${ }^{144} \mathrm{Ba}$ (for the heavy one).

In figure 5 (a) the capture, quasifission, fusion, and fast fission excitation functions are presented. Figure 5 (b) shows the $P_{\mathrm{CN}}$ fusion probability in the same $E_{\text {c.m. }}$ energy range. This quantity is determined as a ratio of complete fusion cross section to the capture cross section: $P_{\mathrm{CN}}\left(E_{\text {c.m. }}\right)=$ $\sigma_{\text {fus }}\left(E_{\text {c.m. }}\right) / \sigma_{\text {cap }}\left(E_{\text {c.m. }}\right)$. The results demonstrate that, also in this case of almost mass symmetric reaction, the quasifission process strongly dominates over complete fusion and the $P_{\mathrm{CN}}$ fusion probability ranges between $10^{-11}-10^{-4}$
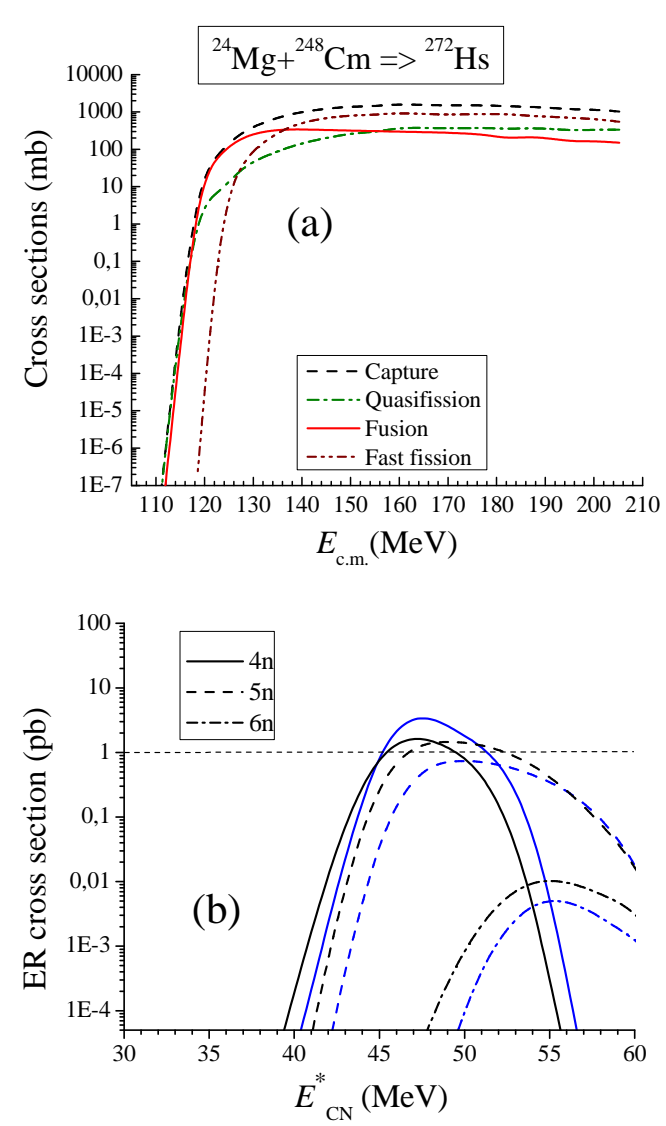

Fig. 4. The capture, quasifission, fusion and fast fission cross sections (panel (a)) versus the $E_{\text {c.m. }}$ energy, and the individual evaporation residue cross sections (panel (b)) versus the $E_{\mathrm{CN}}^{*}$ excitation energy of $\mathrm{CN}$, for the ${ }^{24} \mathrm{Mg}+{ }^{248} \mathrm{Cm}$ reaction. In panel (b) are reported the calculation when the masses and barriers of Ref. [23] (black lines) or Refs. [24,25] (blue lines) are used.

in the explored energy range. Moreover, in figures 6 (a) and 6 (b), we report the angular momentum distribution of the capture and complete fusion stages of the ${ }^{108} \mathrm{Mo}+{ }^{144} \mathrm{Ba}$ reaction, respectively. As one can see, the capture is strongly contributed by the $50-100 \hbar$ orbital angular momentum range, while the largest contribution of complete fusion is peaked at around $25 \hbar$. The results presented in figures 5 and 6 showed smallness of the fusion probability of two colliding nuclei which are ${ }^{108} \mathrm{Mo}$ and ${ }^{144} \mathrm{Ba}$ being fission products of ${ }^{252} \mathrm{Cf}$ observed with largest probability. So, we can conclude that complete fusion can not be considered as inverse channel to fission of compound nucleus having large probability. Therefore, it is impossible to believe to the statement that the fusion process can be considered as the inverse process of fission. Due to mentioned above the dependence of the entrance channel dynamics on the characteristics of each beam-target combination, it is impossible to obtain the same $\mathrm{CN}$ (with the same $Z, A$, and $E_{\mathrm{CN}}^{*}$ excitation energy) with the same dynamic properties (shapes and spin distribution mainly) by two very different reactions. The DNS model allows us to consider an influence of the microscopic structure properties (for example shell effects) of reactants to the dynamic properties of being formed rotating and excited nucleus. 


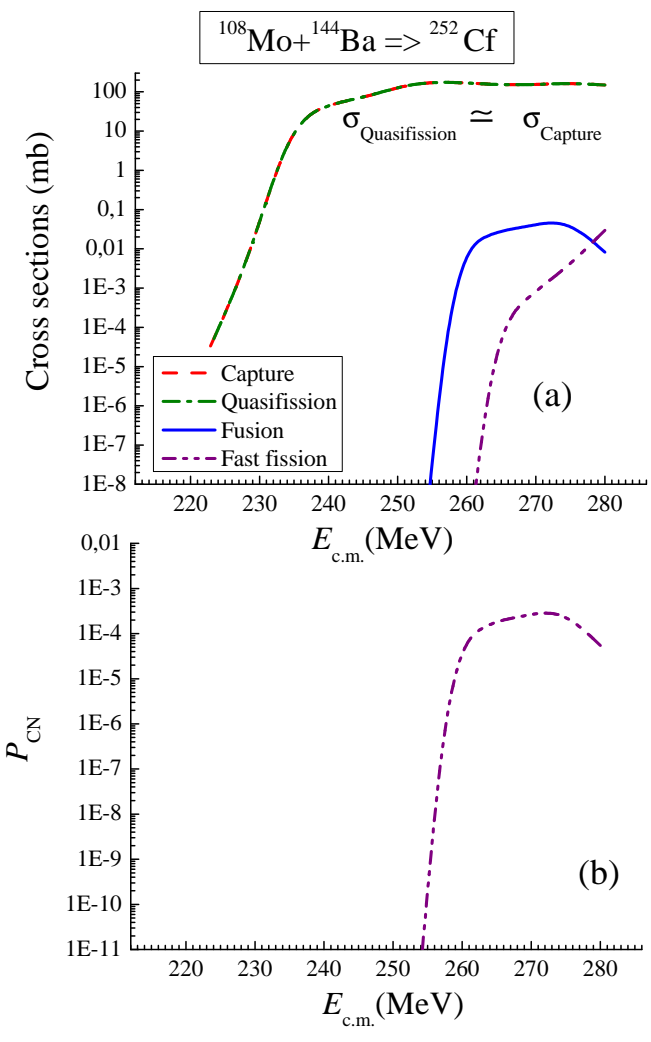

Fig. 5. Cross sections (panel (a)) and $P_{\mathrm{CN}}$ fusion probability (panel (b)) versus the $E_{\text {c.m. }}$ energy, for the ${ }^{108} \mathrm{Mo}+{ }^{144} \mathrm{Ba}$ reaction.
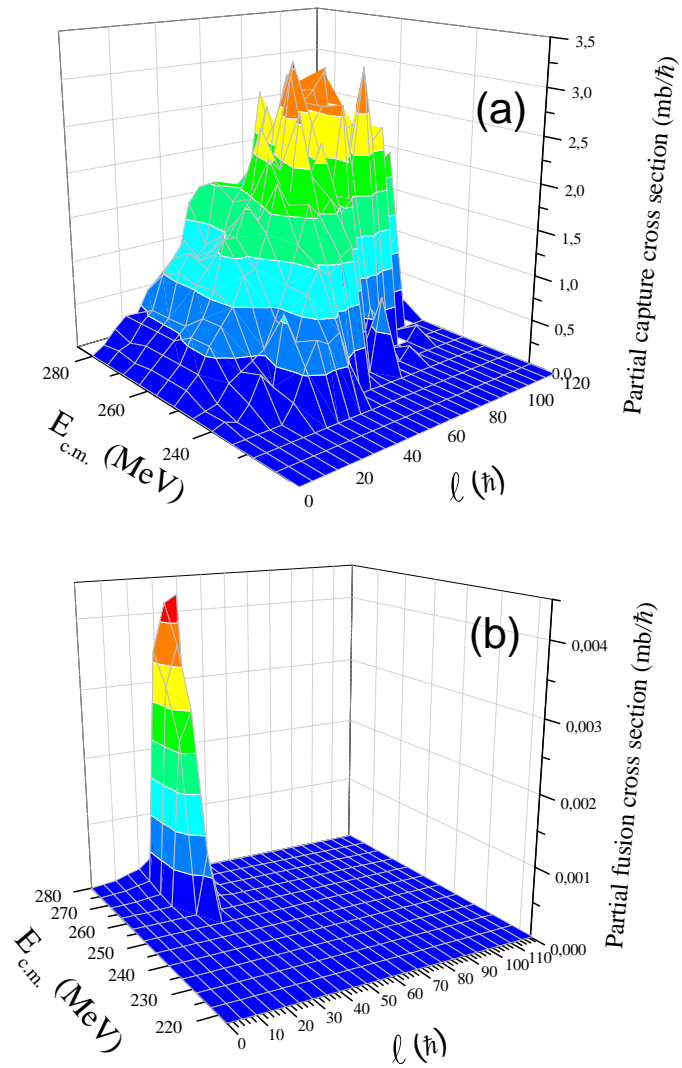

Fig. 6. Spin distribution of capture (panel (a)) and fusion (panel (b)) cross sections as a function of $E_{\text {c.m. }}$ energy, for the ${ }^{108} \mathrm{Mo}+{ }^{144} \mathrm{Ba}$ reaction.

\section{Investigation on the superheavy nuclei and perspectives for heavier superheavy elements}

In order to estimate the realistic possibilities to synthesize superheavy elements by massive nuclei reactions, we calculated the ER cross sections for a set of reactions forming fissile compound nuclei with $Z \geq 100$ at the same excitation energy $\left(E_{\mathrm{CN}}^{*} \simeq 37 \mathrm{MeV}\right)$. In Table 1 we present the set of studied reactions by various entrance channels with different charge (mass) asymmetry parameters.

Table 1. The listed reactions are reported as a function of the charge $Z_{\mathrm{CN}}$ of $\mathrm{CN}$ (if it can be reached), and the parameter $z=$ $\frac{Z_{1} \times Z_{2}}{A_{1}^{1 / 3}+A_{2}^{1 / 3}}$ representing the Coulomb barrier of reacting nuclei in the entrance channel.

\begin{tabular}{|c|c|c||c|c|c|}
\hline Reaction & $Z_{\mathrm{CN}}$ & $z$ & Reaction & $Z_{\mathrm{CN}}$ & $z$ \\
\hline${ }^{16} \mathrm{O}+{ }^{238} \mathrm{U}$ & 100 & 84 & ${ }^{86} \mathrm{Kr}+{ }^{208} \mathrm{~Pb}$ & 118 & 286 \\
\hline${ }^{48} \mathrm{Ca}+{ }^{208} \mathrm{~Pb}$ & 102 & 172 & ${ }^{132} \mathrm{Sn}+{ }^{174} \mathrm{Yb}$ & 120 & 328 \\
\hline${ }^{50} \mathrm{Ti}+{ }^{208} \mathrm{~Pb}$ & 104 & 188 & ${ }^{64} \mathrm{Ni}+{ }^{238} \mathrm{U}$ & 120 & 253 \\
\hline${ }^{136} \mathrm{Xe}+{ }^{136} \mathrm{Xe}$ & 108 & 284 & ${ }^{58} \mathrm{Fe}+{ }^{244} \mathrm{Pu}$ & 120 & 242 \\
\hline${ }^{58} \mathrm{Fe}+{ }^{208} \mathrm{~Pb}$ & 108 & 218 & ${ }^{54} \mathrm{Cr}+{ }^{248} \mathrm{Cm}$ & 120 & 229 \\
\hline${ }^{48} \mathrm{Ca}+{ }^{226} \mathrm{Ra}$ & 108 & 181 & ${ }^{132} \mathrm{Sn}+{ }^{176} \mathrm{Hf}$ & 122 & 337 \\
\hline${ }^{26} \mathrm{Mg}+{ }^{248} \mathrm{Cm}$ & 108 & 125 & ${ }^{54} \mathrm{Cr}+{ }^{249} \mathrm{Cf}$ & 122 & 234 \\
\hline${ }^{48} \mathrm{Ca}+{ }^{243} \mathrm{Am}$ & 115 & 193 & ${ }^{132} \mathrm{Sn}+{ }^{186} \mathrm{~W}$ & 124 & 343 \\
\hline${ }^{48} \mathrm{Ca}+{ }^{248} \mathrm{Cm}$ & 116 & 194 & ${ }^{58} \mathrm{Fe}+{ }^{249} \mathrm{Cf}$ & 124 & 251 \\
\hline${ }^{48} \mathrm{Ca}+{ }^{248} \mathrm{Bk}$ & 117 & 196 & ${ }^{84} \mathrm{Kr}+{ }^{232} \mathrm{Th}$ & 126 & 307 \\
\hline${ }^{48} \mathrm{Ca}+{ }^{249} \mathrm{Cf}$ & 118 & 198 & ${ }^{64} \mathrm{Ni}+{ }^{249} \mathrm{Cf}$ & 126 & 267 \\
\hline
\end{tabular}

It is interesting to observe and analyze the overall trend of the fusion probability $P_{\mathrm{CN}}$ and the evaporation residue yields in different reactions as a function of the charge $Z_{\mathrm{CN}}$ of $\mathrm{CN}$ and of the parameter $z=\frac{Z_{1} \times Z_{2}}{A_{1}^{1 / 3}+A_{2}^{1 / 3}}$ (related to the Coulomb barrier in the entrance channel) in order to draw some useful indications on the reactions which may lead to synthesize heavy nuclei with $Z_{\mathrm{CN}} \geq 100$ and superheavy elements with $Z_{\mathrm{CN}} \geq 120$.

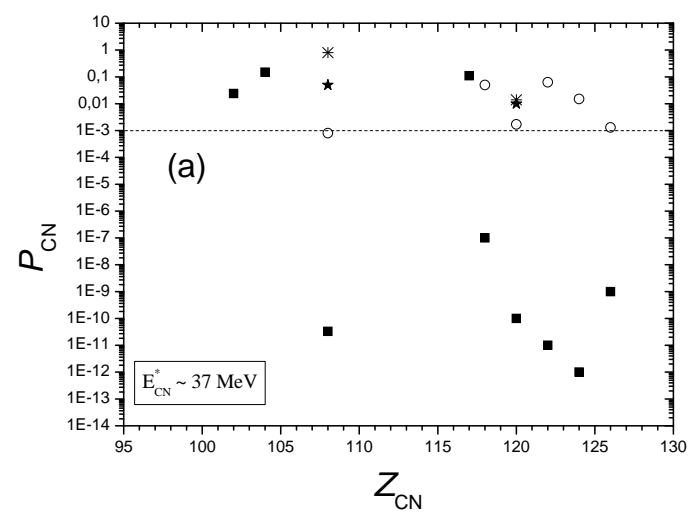

Fig. 7. Fusion probability $P_{\mathrm{CN}}$ calculated at the same excitation energy $E_{\mathrm{CN}}^{*} \simeq 37 \mathrm{MeV}$ versus charge $Z_{\mathrm{CN}}$ for the reactions listed in Table 1.

Figure 7 shows the fusion probability $P_{\mathrm{CN}}$ for the reactions listed in Table 1 as a function of the charge $Z_{\mathrm{CN}}$ at excitation energy $E_{\mathrm{CN}}^{*} \simeq 37 \mathrm{MeV}$. As one can see in this 


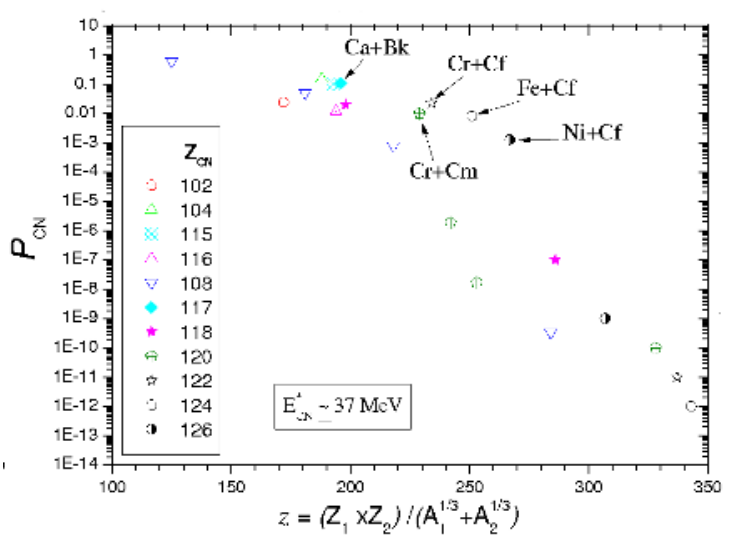

Fig. 8. Fusion probability $P_{\mathrm{CN}}$ versus the parameter $z$ (representing the Coulomb barrier of reacting nuclei in the entrance channel) for many reactions with charge of $\mathrm{CN} Z_{\mathrm{CN}}$ included in the $Z_{\mathrm{CN}}=100-126$ range.

figure that $P_{\mathrm{CN}}$ slowly decreases by increasing $Z_{\mathrm{CN}}$ but it decreases strongly for more symmetric reactions in the entrance channel leading to the same $Z_{\mathrm{CN}}$. The $P_{\mathrm{CN}}$ values decrease for less asymmetric reactions. The trend of $P_{\mathrm{CN}}$ appears more clear for the same investigated reactions if we present $P_{\mathrm{CN}}$ as a function of the parameter $z=\frac{Z_{1} \times Z_{2}}{A_{1}^{1 / 3}+A_{2}^{1 / 3}}$ representing the effect of the Coulomb barrier of interacting nuclei in the entrance channel (see figure 8). As figure 8 shows the trend of $P_{\mathrm{CN}}$ at $E_{\mathrm{CN}}^{*} \simeq 37 \mathrm{MeV}$ strongly decreases by increasing the $z$ parameter and by decreasing the charge (mass) asymmetry parameter of reactions in the entrance channel. The hindrance to complete fusion increases for more symmetric reactions and for higher Coulomb barriers of reactions in the entrance channel.

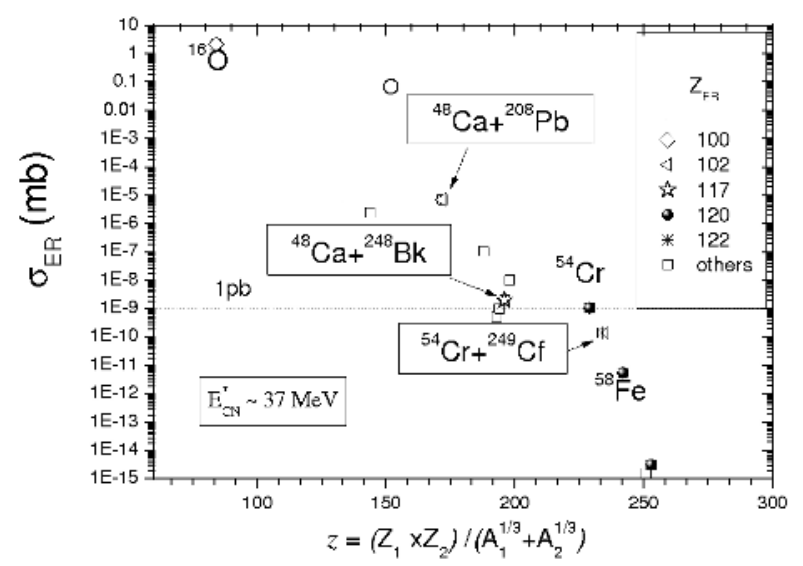

Fig. 9. Evaporation residue cross section $\sigma_{\mathrm{ER}}$ (after neutron emission only) versus the parameter $z$ representing the Coulomb barrier in the entrance channel, for reactions with $Z_{C N}=100-120$.

Figure 9 shows the evaporation residue cross sections, after only neutron emission from $\mathrm{CN}$, obtained for the investigated reactions as a function of the parameter $z$, at $E_{\mathrm{CN}}^{*} \simeq 37 \mathrm{MeV}$. In this figure the horizontal dotted line marks the value of $1 \mathrm{pb}$ for the ER cross section. The evaporation residues after neutron emission only from the de-excitation cascade of $\mathrm{CN}$ can be observed for reactions with parameter $z$ lower than the values of about 200. For values set of parameter $z$ included in the range about 200235 the observation of residues is at limit (or it appears to be a very problematic task) of the current experimental possibilities. For reactions with $z$ higher than 235 it is impossible to observe ER of $\mathrm{CN}$ after neutron emission only.

\section{Reactions leading to compound nuclei with $Z \geq 120$}

The results of calculations obtained for the reactions leading to $\mathrm{CN}$ with $Z=120,122,124$, and 126 , at excitation energy of compound nuclei of about $37 \mathrm{MeV}$ are presented in Table 2.

Table 2. Reactions leading to compound nuclei with $Z_{\mathrm{CN}}=120$, $122,124,126$, as a function of the parameter $z$ representing the Coulomb barrier in the entrance channel. $\sigma_{\mathrm{ER}}$ is the ER cross section after the neutron emission only from the de-excitation cascade of $\mathrm{CN} ; P_{\text {res/cap }}$ is the ratio between the yields of evaporation residue $\sigma_{\mathrm{ER}}$ and the capture $\sigma_{\text {cap }}$.

\begin{tabular}{|c|c|c|c|c|}
\hline Reaction & $Z_{\mathrm{CN}}$ & $z$ & $\sigma_{\mathrm{ER}}(\mathrm{mb})$ & $P_{\text {res/cap }}$ \\
\hline${ }^{54} \mathrm{Cr}+{ }^{248} \mathrm{Cm}$ & 120 & 229 & $1.05 \times 10^{-10}$ & $0.3 \times 10^{-11}$ \\
\hline${ }^{58} \mathrm{Fe}+{ }^{244} \mathrm{Pu}$ & 120 & 242 & $5.4 \times 10^{-12}$ & $0.17 \times 10^{-13}$ \\
\hline${ }^{64} \mathrm{Ni}+{ }^{238} \mathrm{U}$ & 120 & 253 & $3.1 \times 10^{-15}$ & $0.14 \times 10^{-15}$ \\
\hline${ }^{54} \mathrm{Cr}+{ }^{249} \mathrm{Cf}$ & 122 & 234 & $1.4 \times 10^{-10}$ & $0.13 \times 10^{-11}$ \\
\hline${ }^{58} \mathrm{Fe}+{ }^{249} \mathrm{Cf}$ & 124 & 251 & $1.61 \times 10^{-15}$ & $0.18 \times 10^{-16}$ \\
\hline${ }^{64} \mathrm{Ni}+{ }^{249} \mathrm{Cf}$ & 126 & 267 & $4.4 \times 10^{-20}$ & $6.5 \times 10^{-22}$ \\
\hline
\end{tabular}

Figure 10 shows the theoretical results of ER as a function of the parameter $z=\frac{Z_{1} \times Z_{2}}{A_{1}^{1 / 3}+A_{2}^{1 / 3}}$ at $E_{\mathrm{CN}}^{*} \simeq 37$. The dotted line the value of $\sigma_{\mathrm{ER}}$ of $1 \mathrm{pb}$. Our estimations showed that

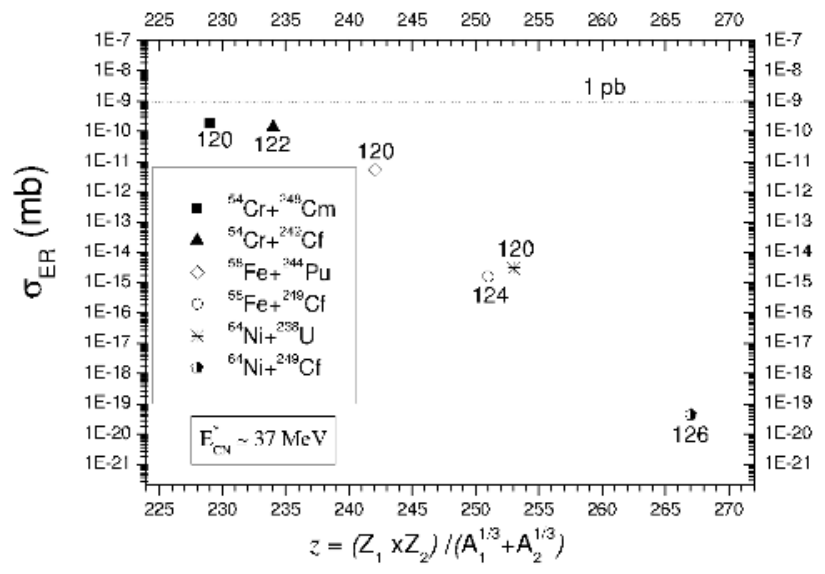

Fig. 10. As figure 9, but for reactions leading to $Z_{\mathrm{CN}}=120-126$.

the superheavy element with $Z=120$ may be observed in reactions having $z$ parameter value lower than 230 . The observation of the superheavy element $Z=122$ in reactions with $z \approx 234$ appears to be a very doubtful venture, while the observations of the superheavy elements with $Z=124$ and 126 in reactions with $z$ of about 251 and 267, respectively, appear as impossible by the current experimental conditions and detecting system of evaporation residues. 


\section{Conclusions}

On the basis of dynamical and statistical calculations we studied a formation of a dinuclear system in the entrance channel and its subsequent evolution to be transformed into compound nucleus in a competition with quasifission. The distribution of the $P_{\mathrm{CN}}$ fusion probability versus the $\mathrm{CN}$ charge $Z_{\mathrm{CN}}$, angular momentum in the entrance channel and $E_{\text {c.m. }}$. energy has been estimated for a wide set of reactions. From the study of such systematics we can understand the effect of the entrance channel mass asymmetry on the fusion probability and evaporation residue yields in many reactions forming various compound nuclei at the same excitation energy $E_{\mathrm{CN}}^{*}$ of about $37 \mathrm{MeV}$.

The trend of $P_{\mathrm{CN}}$ is represented by a slow decrease with the increase of the charge $Z_{\mathrm{CN}}$, a significant decrease of the $P_{\mathrm{CN}}$ values by decrease of the mass (charge) asymmetry parameter of reactants in the entrance channel forming the same $\mathrm{CN}$, and a fast decrease of the $P_{\mathrm{CN}}$ values and ER yields versus the parameter $z=\frac{Z_{1} \times Z_{2}}{A_{1}^{1 / 3}+A_{2}^{1 / 3}}$.

Regarding the results of the investigated reactions leading to the formation of compound nuclei with $Z_{\mathrm{CN}}=120$, 122,124 , and 126 we affirm that there is still possibility to reach and observe the ER of the 120 superheavy element by a reaction with $z$ parameter value of about 230 , while it is a very doubtful venture to synthesize the 122 superheavy element by reactions with $z$ parameter value of about 234 or higher by the current experimental resources and methods of observing evaporation residues.

A possibility to observe evaporation residue of superheavy elements disappears in reactions with $z$ parameter in the entrance channel higher than 240 . Therefore, it is impossible to form the 124 and 126 superheavy nuclei in these kind of reactions.

The quasifission is the main process causing the hindrance to complete fusion and the contribution of this process strongly increases for reactions with large values of $z$ parameter and also with the increase of the colliding energy $E_{\text {c.m. }}^{*}$. The fast fission and fusion-fission are the subsequent hindrances competing with formation of evaporation residues after surviving system against to quasifission. In this context, the mass symmetric or nearly symmetric reactions in the entrance channels do not give a realistic possibility to synthesize superheavy elements, and the use of the ${ }^{132} \mathrm{Sn}$ beam is of scarce usefulness for this kind of reactions.

Consequently, it is an unrealizable dream to think of performing the ${ }^{132} \mathrm{Sn}+{ }^{208} \mathrm{~Pb}$ (with $z=373$ ) and ${ }^{132} \mathrm{Sn}+{ }^{249} \mathrm{Cf}$ (with $z=431$ ) reactions in order to reach the ${ }^{340} 132$ and ${ }^{381} 148$ superheavy elements, respectively, and by the mass symmetric reactions as ${ }^{136} \mathrm{Xe}+{ }^{136} \mathrm{Xe}$ (with $z=184$ ) and ${ }^{139,149} \mathrm{La}+{ }^{139,149} \mathrm{La}$ (with $z=317$ and 306 , respectively) to synthesize heavy and superheavy elements to cause of the absolute dominant contribution of the quasifission process after capture, and the fast fission process presents at stage of the small probable formation of complete fusion.

Moreover, we discussed the role of the entrance channel characteristics and Coulomb forces on the reaction dynamics, complete fusion, and formation of the evaporation residues which are a synthesized superheavy elements. As the example, the evaporation residue cross sections, which were calculated for the ${ }^{136} \mathrm{Xe}+{ }^{136} \mathrm{Xe}$ and ${ }^{24} \mathrm{Mg}+$ ${ }^{248} \mathrm{Cm}$ reactions leading to the same ${ }^{272} \mathrm{Hs} \mathrm{CN}$, have been compared. In addition, we also discussed the reason for which the complete fusion process of two massive reactants (for example in the ${ }^{108} \mathrm{Mo}+{ }^{144} \mathrm{Ba}$ reaction) cannot be considered as the inverse process of fission (for example in the ${ }^{252} \mathrm{Cf}$ spontaneous fission) leading to fragments (for example ${ }^{108} \mathrm{Mo}$ and ${ }^{144} \mathrm{Ba}$ ) that are the same nuclei used in the proposed fusion reaction.

\section{References}

1. K. Siwek-Wilczynska, et al., Int. J. Mod. Phys. E 16, 493 (2007)

2. R. Smolanczuk, Phys. Rev. C 63, 044607 (2001)

3. V. I. Zagrebaev and W. Greiner, Nucl. Phys. A 787, 363 (2007)

4. Yu. Ts. Oganessian, et al., Phys. Rev. C 74, 044602 (2006)

5. V. I. Zagrebaev, A. V. Karpov, and W. Greiner, Phys. Rev. C 85, 014608 (2012)

6. N. A. Antonenko, et al., Phys. Lett. B 319, 425 (1993); Phys. Rev. C 51, 2635 (1995)

7. G. Fazio et al., Phys.Rev. C 72, 064614 (2005)

8. A. Nasirov, et al., Nucl. Phys. A 759, 342 (2005)

9. G. Fazio, et al., Eur. Phys. J. A 19, 89 (2004)

10. G. Giardina, S. Hofmann, A. I. Muminov, A. K. Nasirov, Eur. Phys. J. A 8, 205 (2000)

11. G. Fazio, et al., J. Phys. Soc. Japan 72, 2509 (2003)

12. A. K. Nasirov, et al., Phys. Rev. C 79, 024606 (2009)

13. G. Fazio, et al., J. Phys. Soc. Japan 77, 124201 (2008)

14. G. Fazio, et al., Mod. Phys. Lett. A 20, 391 (2005)

15. A. D'Arrigo, et al., Phys. Rev. C 46, 1437 (1992)

16. A. D'Arrigo, et al., J. Phys. G 20, 365 (1994)

17. R. N. Sagaidak, et al., J. Phys. G 24, 611 (1998)

18. G. Giardina, et al., J. Phys. CS 282, 012006 (2011)

19. A. K. Nasirov, et al., Phys. Rev. C 79, 024606 (2010)

20. H. Q. Zhang, et al., Phys. Rev. C 81, 034611 (2010)

21. G. Fazio, et al., Eur. Phys. J. A 22, 75 (2004)

22. G. Fazio, et al., Phys. Rev. C 72, 064614 (2005)

23. P. Möller and J. R. Nix, J. Phys. G: Nucl. Part. Phys. 20, 1681 (1994)

24. I. Muntian, Z. Patyk, and A. Sobiczewski, Phys. Atom. Nucl. 66, 1015 (2003)

25. M. Kowal, P. Jachimowicz, and A. Sobiczewski, Phys. Rev. C 82, 014303 (2010) 